\title{
reciamuc
}

Revista cientifica de investigación actualización del mundo de las ciencias

\author{
Gema Nathalye Rodríguez Chica a; Marlon Gastón Ramírez Saverio ${ }^{\text {b; }}$ Stefani \\ Elizabeth Sánchez Alcívar ${ }^{c}$; Jefferson Saúl Triviño Sánchez ${ }^{\mathrm{d}}$
}

Tratamiento en pacientes con cefalea postpunción dural

Treatment in patients with dural post-puncture headache

Revista Científica de Investigación actualización del mundo de las Ciencias. Vol. 3 núm., 3, julio, ISSN: 2588-0748, 2018, pp. 881-901

DOI: $10.26820 /$ reciamuc/3.(3).julio.2019.881-901

\section{URL: http://reciamuc.com/index.php/RECIAMUC/article/view/308}

Código UNESCO: 3205 Medicina Interna

Tipo de Investigación: Artículo de Revisión

(C) RECIAMUC; Editorial Saberes del Conocimiento, 2019

Recibido: 28/04/2019

Aceptado: 19/05/2019

Publicado: 01/07/2019

Correspondencia: gemanathalye@gmail.com
a. Médico; Investigador Independiente; Guayaquil, Ecuador; gemanathalye @ gmail.com
b. Médico; Investigador Independiente; Guayaquil, Ecuador; mramirezsaverio@gmail.com
c. Médico; Investigador Independiente; Guayaquil, Ecuador; ssanchezalcivar89@ gmail.com
d. Médico; Investigador Independiente; Guayaquil, Ecuador; jeff_sts2@ hotmail.com 


\section{Tratamiento en pacientes con cefalea postpunción dural}

Vol. 3, núm. 3., (2019)

Gema Nathalye Rodríguez Chica; Marlon Gastón Ramírez Saverio; Stefani Elizabeth Sánchez Alcívar; Jefferson Saúl Triviño Sánchez

\section{RESUMEN}

Después de leer este artículo, los lectores deben ser capaces de reconocer la post punción dural de cabeza, comprender su mecanismo y criterios de diagnóstico, evaluar las diferentes opciones de tratamiento disponibles, y estar familiarizado con una opción de tratamiento novedoso. La cefalea pos punción dural es la complicación grave más común que resulta de la punción lumbar y anestesia epidural o espinal. El síndrome se caracteriza por dolor de cabeza grave que se produce dentro de las 48 horas siguientes a la punción, situada en el frontal y / o la región occipital. Dos mecanismos posibles son hipotetizados como responsables de este síndrome; fugas de líquido cefalorraquídeo y neumocefalo. Múltiples métodos de tratamiento se han aplicado con amplios resultados. Se postulan que la terapia de oxígeno hiperbárico puede ser utilizado para tratar el dolor de cabeza posterior a la punción dural. El fundamento para el tratamiento es doble: aumento de la proliferación fi-broblastos en el sitio de la punción dural para facilitar el cierre más rápido de la rotura y la compresión de burbujas de aire en caso de neumoencéfalo según la ley de Boyle. También afirmamos que la terapia con oxígeno hiperbárico se debe considerar un tratamiento de profilaxis láctico, si se sospecha un desgarro de la duramadre.

Palabras Claves: Punción Dural de Cabeza; Punción Lumbar; Anestesia Epidural; Anestesia Espinal; Dolor de Cabeza; Terapia de Oxígeno Hiperbárico. 


\title{
Tratamiento en pacientes con cefalea postpunción dural
}

Vol. 3, núm. 3., (2019)

Gema Nathalye Rodríguez Chica; Marlon Gastón Ramírez Saverio; Stefani Elizabeth Sánchez

Alcívar; Jefferson Saúl Triviño Sánchez

\begin{abstract}
After reading this article, readers should be able to recognize the post dural puncture of the head, understand its mechanism and diagnostic criteria, evaluate the different treatment options available, and be familiar with a novel treatment option. Post-dural puncture headache is the most common serious complication that results from lumbar puncture and epidural or spinal anesthesia. The syndrome is characterized by severe headache that occurs within 48 hours after the puncture, located in the frontal and / or occipital region. Two possible mechanisms are hypothesized to be responsible for this syndrome; leakage of cerebrospinal fluid and pneumocephalus. Multiple treatment methods have been applied with extensive results. It is postulated that hyperbaric oxygen therapy can be used to treat headache after dural puncture. The rationale for the treatment is twofold: increased proliferation of fibroblasts at the site of the dural puncture to facilitate faster closure of the rupture and compression of air bubbles in the case of pneumocephalus according to Boyle's law. We also affirm that hyperbaric oxygen therapy should be considered a lactic prophylaxis treatment, if a tear of the dura is suspected.
\end{abstract}

Key Words: Head Dural Puncture; Lumbar Puncture; Epidural Anesthesia; Spinal Anesthesia; Headache; Hyperbaric Oxygen Therapy. 


\section{Tratamiento en pacientes con cefalea postpunción dural}

Vol. 3, núm. 3., (2019)

Gema Nathalye Rodríguez Chica; Marlon Gastón Ramírez Saverio; Stefani Elizabeth Sánchez Alcívar; Jefferson Saúl Triviño Sánchez

\section{Introducción.}

La anestesia espinal fue desarrollada al final del siglo 19. Quincke y Wynterfueron los primeros en realizar la punción lumbar (PL) en 1891 para aliviar el aumento de la presión intracraneal en niños con meningitis tuberculosa (Qunicke, 1909). Cuatro años más tarde (1895), Corningsugirio anestesia espinal local con la cocaína para tratar a un hombre con masturbación habitual (Gorelick \& Zych, 1987). En 1898 Bier sufrió y fue el primero en informar cefalea post punción dural Dolor (CPPD) después de la inyección de cocaína en el espacio subaracnoideo de siete pacientes incluidos él y su asistente Hildebrandt. Bier también propuso que la fuga continua de fluido cerebrospinal (CSF) era la causa del dolor de cabeza. En la anestesia moderna, CPPD se informa, es la complicación más frecuente de bloques espinal y epidural. A pesar de la prevalencia del síndrome, se sabe poco sobre su fisiopatología y tratamientos más aceptados no están basadas en la evidencia.

En el siguiente artículo se intenta reunir toda la información sobre las opciones de tratamiento disponibles en la actualidad. El propósito del artículo es dar a conocer las técnicas de tratamiento para CPPD, entre ellas la terapia de oxígeno hiperbárico $(\mathrm{TOH})$. Se cree que una vez que se sospecha de una punción accidental de la duramadre, TOH debe iniciarse de inmediato para minimizar el riesgo de desarrollo de la CPPD. El fundamento para el tratamiento es de proliferación de fibroblastos en el sitio de la punción, el mismo mecanismo que se aplica para las heridas crónicas.

Aunque la CPPD generalmente se resuelve de manera espontánea, tiene el potencial de causar una morbilidad significativa en los pacientes obstétricos. También puede interferir con la 


\section{Tratamiento en pacientes con cefalea postpunción dural}

Vol. 3, núm. 3., (2019)

Gema Nathalye Rodríguez Chica; Marlon Gastón Ramírez Saverio; Stefani Elizabeth Sánchez Alcívar; Jefferson Saúl Triviño Sánchez

capacidad de la madre para cuidarse a sí misma oa su bebé, y puede prolongar la duración de la estadía en el hospital o convertirse en un dolor de cabeza crónico. En un estudio retrospectivo de casos y controles, (Webb, y otros, 2012) informaron que el dolor de cabeza crónico se presentó en el $28 \%$ de las mujeres obstétricas después de una punción dural inadvertida con una aguja Tuohy de calibre 17. La prevención de la CPPD debe ser el objetivo principal de los clínicos que tratan con esta población. De hecho, la atención adecuada a los factores relacionados con el procedimiento puede reducir significativamente su incidencia. Ocasionalmente, la punción dural inadvertida y la CPPD son complicaciones inevitables. Por lo tanto, los anestesiólogos deben estar familiarizados con las modalidades de tratamiento y la prevención. Esta revisión resume los conceptos actuales relacionados con el diagnóstico, la fisiopatología, los factores de riesgo, la prevención y el tratamiento de la CPPD.

\section{Metodología.}

Para el desarrollo de este proceso investigativo, se plantea como metodología la encaminada hacia una orientación científica particular que se encuentra determinada por la necesidad de indagar en forma precisa y coherente una situación, en tal sentido (Davila, 2015) define la metodología "como aquellos pasos previos que son seleccionados por el investigador para lograr resultados favorables que le ayuden a plantear nuevas ideas”. (p.66)

Lo citado por el autor, lleva a entender que el desarrollo de la acción investigativa busca simplemente coordinar acciones enmarcadas en una revisión bibliográfica con el fin de complementar ideas previas relacionadas a los tratamientos a pacientes con cefalea post punción 


\section{Tratamiento en pacientes con cefalea postpunción dural}

Vol. 3, núm. 3., (2019)

Gema Nathalye Rodríguez Chica; Marlon Gastón Ramírez Saverio; Stefani Elizabeth Sánchez Alcívar; Jefferson Saúl Triviño Sánchez

dural a través de una revisión de literatura, para así finalmente elaborar un cuerpo de consideraciones generales que ayuden a ampliar el interés propuesto.

\section{Tipo de Investigación}

Dentro de toda práctica investigativa, se precisan acciones de carácter metodológico mediante las cuales, se logra conocer y proyectar los eventos posibles que la determinan, así como las características que hacen del acto científico un proceso interactivo ajustado a una realidad posible de ser interpretada. En este sentido, se puede decir, que la presente investigación corresponde al tipo documental, definido por Castro (2016), "se ocupa del estudio de problemas planteados a nivel teórico, la información requerida para abordarlos se encuentra básicamente en materiales impresos, audiovisuales y /o electrónicos”. (p.41).

En consideración a esta definición, la orientación metodológica permitió la oportunidad de cumplir con una serie de actividades inherentes a la revisión y lectura de diversos documentos donde se encontraron ideas explicitas relacionadas con los tópicos encargados de identificar a cada característica insertada en el estudio. Por lo tanto, se realizaron continuas interpretaciones con el claro propósito de revisar aquellas apreciaciones o investigaciones propuestas por diferentes investigadores relacionadas con el tema de interés, para luego dar la respectiva argumentación a los planteamientos, en función a las necesidades encontradas en la indagación.

\section{Fuentes Documentales}

El análisis correspondiente a las características que predomina en el tema seleccionado, llevan a incluir diferentes fuentes documentales encargadas de darle el respectivo apoyo y en ese 


\section{Tratamiento en pacientes con cefalea postpunción dural}

Vol. 3, núm. 3., (2019)

Gema Nathalye Rodríguez Chica; Marlon Gastón Ramírez Saverio; Stefani Elizabeth Sánchez Alcívar; Jefferson Saúl Triviño Sánchez

sentido cumplir con la valoración de los hechos a fin de generar nuevos criterios que sirven de referencia a otros procesos investigativos. Para (CASTRO, 2016) las fuentes documentales incorporadas en la investigación documental o bibliográfica, "representa la suma de materiales sistemáticos que son revisados en forma rigurosa y profunda para llegar a un análisis del fenómeno". (p.41). Por lo tanto, se procedió a cumplir con la realización de una lectura previa determinada para encontrar aquellos aspectos estrechamente vinculados con el tema, con el fin de explicar mediante un desarrollo las respectivas apreciaciones generales de importancia.

\section{Técnicas para la Recolección de la Información}

La conducción de la investigación para ser realizada en función a las particularidades que determinan a los estudios documentales, tiene como fin el desarrollo de un conjunto de acciones encargadas de llevar a la selección de técnicas estrechamente vinculadas con las características del estudio. En tal sentido, (Bolívar, 2015), refiere, que es "una técnica particular para aportar ayuda a los procedimientos de selección de las ideas primarias y secundarias”. (p. 71).

Por ello, se procedió a la utilización del subrayado, resúmenes, fichaje, como parte básica para la revisión y selección de los documentos que presentan el contenido teórico. Es decir, que mediante la aplicación de estas técnicas se pudo llegar a recoger informaciones en cuanto a la revisión bibliográfica de los diversos elementos encargados de orientar el proceso de investigación. Tal como lo expresa, (Bolívar, 2015) "las técnicas documentales proporcionan las herramientas esenciales y determinantes para responder a los objetivos formulados y llegar a resultados efectivos" (p. 58). Es decir, para responder con eficiencia a las necesidades investigativas, se introdujeron como técnica de recolección el método inductivo, que hizo posible 


\section{Tratamiento en pacientes con cefalea postpunción dural}

Vol. 3, núm. 3., (2019)

Gema Nathalye Rodríguez Chica; Marlon Gastón Ramírez Saverio; Stefani Elizabeth Sánchez Alcívar; Jefferson Saúl Triviño Sánchez

llevar a cabo una valoración de los hechos de forma particular para llegar a la explicación desde una visión general.

Asimismo, se emplearon las técnicas de análisis de información para la realización de la investigación que fue ejecutada bajo la dinámica de aplicar diversos elementos encargados de determinar el camino a recorrer por el estudio, según, (Bolívar, 2015) las técnicas de procesamiento de datos en los estudios documentales "son las encargadas de ofrecer al investigador la visión o pasos que debe cumplir durante su ejercicio, cada una de ellas debe estar en correspondencia con el nivel a emplear" (p. 123). Esto indica, que para llevar a cabo el procesamiento de los datos obtenidos una vez aplicado las técnicas seleccionadas, tales como: fichas de resumen, textual, registros descriptivos entre otros, los mismos se deben ajustar al nivel que ha sido seleccionado.

\section{Resultados.}

Anatomía, Histología y Fisiopatología de la punción dural

\section{Las meninges}

La médula espinal está rodeada por las tres meninges; duramadre, aracnoides y piamadre. Estas membranas rodean, apoyan y protegen la médula espinal y las raíces nerviosas espinales, incluyendo los de la cola de caballo, y contienen el CSF en la que se suspenden estas estructuras. La duramadre espinal; un tejido conectivo de colágeno vascularizado densa con algunas fibras elásticas, es la membrana más externa que cubre la médula espinal. Históricamente se describe como una estructura orientada longitudinalmente que fue confirmada más tarde por estudios 


\section{Tratamiento en pacientes con cefalea postpunción dural}

Vol. 3, núm. 3., (2019)

Gema Nathalye Rodríguez Chica; Marlon Gastón Ramírez Saverio; Stefani Elizabeth Sánchez Alcívar; Jefferson Saúl Triviño Sánchez

histológicos (Patin, Eckstein, Harum, \& Pallares, 1993). Las relativamente grandes distancias moleculares entre las fibras permiten el movimiento de drogas y de fluido. La duramadre de la médula se separa del hueso periostio cubierto y los ligamentos que forman las paredes del canal vertebral por el espacio epidural. La duramadre espinal forma el saco dural espinal, una vaina tubular larga dentro del canal vertebral que va desde el foramen magnum hasta el cóccix. La aracnoides espinal es una membrana delicada, avascular compuesta de fibroblastos, colágeno y fibras elásticas que las líneas del saco dural espinal y sus vainas de las raíces de la duramadre. En contraste con la duramadre, la aracnoides consta de hileras de células unidas por uniones estrechas y uniones de oclusión. Este fenómeno se traduce en la impedancia de paso del fármaco en el CSF y la contención de CSF en el espacio subaracnoideo que encierra el espacio subaracnoideo CSF lleno que contiene la médula espinal, las raíces nerviosas espinales y ganglios espinales. La aracnoides espinal no se adjunta a la duramadre de la médula, pero se mantiene contra su superficie interior por la presión del CSF. En una punción espinal lumbar, los traversos de aguja de la duramadre de la médula y la aracnoides simultáneamente. Su aposición es la interfaz dura aracnoides, a menudo denominada erróneamente como el "espacio subdural". No hay espacio real que se produce de forma natural en este sitio; es, más bien, una capa de células débil. La aracnoides espinal está separada de la piamadre en la superficie de la médula espinal por el espacio subaracnoideaque contiene CSF. La piamadre espinal, la membrana más interna de recubrimiento de la médula espinal, es el tejido conectivo laxo delgada y transparente que sigue de cerca todas las características de la superficie de la médula espinal (Moore, Agur, \& Dalley, 2013). 


\section{Tratamiento en pacientes con cefalea postpunción dural}

Vol. 3, núm. 3., (2019)

Gema Nathalye Rodríguez Chica; Marlon Gastón Ramírez Saverio; Stefani Elizabeth Sánchez Alcívar; Jefferson Saúl Triviño Sánchez

\section{Fluido cerebroespinal}

El cerebro produce aproximadamente $500 \mathrm{ml}$ de líquido cefalorraquídeo por día $(0,35 \mathrm{ml}$ / min). Este líquido se reabsorbe constantemente, por lo que tan sólo 100 - 160mlestán presente en un momento dado. Las células ependimarias del plexo coroideo producen más de dos tercios de CSF.

La punción dural y la pérdida de CSF-La "Teoría de fugas"

La experiencia de neurocirugía de la perforación de la duramadre es que incluso perforaciones menores deben ser cerradas, ya sea directamente o a través de la aplicación de material de injerto dural sintético o biológico, de lo contrario una fuga CSF continua y se pueden producir adherencias o infección (Turnbull \& Shepherd, 2003). Tras la perforación la pérdida de LCR duramadre se produce. La pérdida excesiva CSF puede llevar a la hipotensión intracraneal (ICH), que es un mecanismo posible para el desarrollo de CPPD. Una posible explicación para el dolor de cabeza es a través de la doctrina de Monro-Kelliedonde la disminución en el líquido CSF conduce a un aumento compensatorio en el volumen sanguíneo. Por tanto, el dolor de cabeza es causado por venodilatación. Los avances en neuroimagen han mejorado la capacidad de correlacionar la pérdida de CSF para el diagnóstico dela ICH y CPPD.

La punción dural y Neumoencéfalo-La "Teoría de aire"

Neumoencéfalo se define como una colección patológica de gas dentro de la cavidad craneal. La mayoría de los casos subsiguientes a técnicas epidurales se han asociado con la pérdida de resistencia Technique (LOR). La técnica LOR es ampliamente utilizado ya sea con 


\section{Tratamiento en pacientes con cefalea postpunción dural}

Vol. 3, núm. 3., (2019)

Gema Nathalye Rodríguez Chica; Marlon Gastón Ramírez Saverio; Stefani Elizabeth Sánchez Alcívar; Jefferson Saúl Triviño Sánchez

aire o solución salina para identificar el espacio epidural. Neumoencefalo es una complicación rara de inadvertida punción dural y la inyección de aire en el espacio subaracnoideo o subdural. Se supone que el dolor de cabeza sintomático surge de la irritación de las raíces espinales por el aire en el paciente sentado. El diagnóstico se puede hacer mediante técnicas de imagen como la tomografía computarizada, resonancia magnética o un XR del cráneo que muestra aire atrapada en la columna vertebral y el cerebro (Moayeri, Henson,, Schaefer, \& Zervas, 1998).

\section{Características clínicas}

Según la Sociedad Internacional de Cefaleas (Society., 2001)hay cuatro criterios para CPPD que incluyen un dolor de cabeza que se desarrolla dentro de 5 días después de la punción dural, empeora dentro de los 15 minutos después de estar sentado o de pie y mejora dentro de los 15 minutos después de acostarse, acompañado por al menos uno de los siguientessintomas: rigidez de nuca, náuseas, fotofobia, tinnitus y hipoacusia, y se resuelve ya sea espontáneamente dentro de una semana o dentro de 48 horas después del tratamiento eficaz de la fuga de fluido espinal. El dolor se agrava con la posición vertical y mejora con el tumbado. Cualquier movimiento que aumenta la presión intracraneal (tal como toser, estornudar, esfuerzos o compresión ocular) puede exacerbar los síntomas. Los signos que se pueden encontrar en el examen físico del paciente incluyen el signo Gutsche: la aplicación de presión manual firme alrededor del abdomen del paciente sentado produce un alivio transitorio. La rigidez de nuca también puede ser vista. Otros pacientes pueden tener una diplopía parálisis del nervio craneal. 


\section{Tratamiento en pacientes con cefalea postpunción dural}

Vol. 3, núm. 3., (2019)

Gema Nathalye Rodríguez Chica; Marlon Gastón Ramírez Saverio; Stefani Elizabeth Sánchez Alcívar; Jefferson Saúl Triviño Sánchez

\section{Factores que influyen en la incidencia}

Factores de riesgo comunes de CPPD son el sexo femenino, en especial las mujeres durante el embarazo, grupos de edad de 20 - 40, una historia previa de CPPD o dolor de cabeza, y un índice de masa corporal más bajo. Las mujeres más jóvenes se mostraron tener un mayor riesgo de CPPD debido a su elasticidad de la fibra que mantiene un defecto dural patentes en comparación con disminución de la elasticidad en una edad más avanzada (Evans, 1998). Se encontró que los fumadores de cigarrillos al tener una menor incidencia de CPPD, posiblemente debido a las propiedades de coágulos promoción del consumo de tabaco que facilitan la oclusión de la punción dural. La experiencia del médico que realiza el procedimiento se mostró a ser irrelevante en una serie de 501 pacientes con CPPD, sin embargo, bajo el conocimiento actual que es lógico pensar que cuanto mayor es el número de intentos de punción dural, mayor será la probabilidad de daño a la punción dural, fístula de líquido cefalorraquídeo y CPPD. No hay ningún estudio que examinó disponible esta relación hasta la fecha.

\section{Factores de riesgo y prevención}

La literatura indica claramente que hay varios factores de riesgo que pueden aumentar el riesgo de CPPD y la manera de evitarlos.

\section{Tamaño de la aguja}

Cuanto menor sea el tamaño de la aguja más pequeño es el defecto dural y por lo tanto menor es la incidencia de CPPD. (Dieterich \& Perkin, 1996) encontraron que la incidencia de 


\section{Tratamiento en pacientes con cefalea postpunción dural}

Vol. 3, núm. 3., (2019)

Gema Nathalye Rodríguez Chica; Marlon Gastón Ramírez Saverio; Stefani Elizabeth Sánchez Alcívar; Jefferson Saúl Triviño Sánchez

CPPD era mucho menor con el uso de 24 - 27 agujas de calibre (5\% - 12\%) en comparación con 20 - 22 agujas de calibre $(20 \%-40 \%)$.

\section{Prevención}

Un gran meta-análisis se llevó a cabo en 2012 por (Bradbury, Singh, Badder, Wakely, \& Jones, 2013).Se realizaron búsquedas de ensayos clínicos aleatorios (ECA) de parturientas que tienen las epidurales de mano de obra, en la que la intervención estudiada podría afectar plausiblemente punción accidental de la duramadre (ADP) o CPPD, y se registró la incidencia de al menos uno de estos. Se incluyeron Cuarenta ECA $(n=11.536$ inserciones epidural), el estudio de la médula epidural combinadas (CSE), pérdida de medio resistencia, parches profilácticos de sangre epidural, orientación del bisel de la aguja, la inserción guiada por ultra-sonido, morfina epidural, agujas Sprotteespecial, la inserción acústica guiada, la administración de cosyntropin, y la analgesia espinal continua. Cinco me-thods se muestran para reducir la CPPD: parche de sangre profiláctico, orientación lateral del bisel de la aguja durante la inserción, uso de la nocorte punta de la aguja epidural, epidural morfina y administración de cosinotropin.

\section{Tratamiento}

Después de confirmar el diagnóstico de CPPD, el tratamiento inicial para las primeras 24 - 48 horas debe permanecer conservador e incluir el reposo en cama, analgésicos orales que no degraden la función plaquetaria tales como los opioides y la hidratación para mantener euvolemia. No hay evidencia que la hidratación vigorosa tiene ningún beneficio terapéutico, o que estimula una producción elevada de CSF, sin embargo ningún paciente con CPPD se debe permitir que caiga en deshidratado. Si se ha hecho el diagnóstico de neumoencefalo, no debería 


\section{Tratamiento en pacientes con cefalea postpunción dural}

Vol. 3, núm. 3., (2019)

Gema Nathalye Rodríguez Chica; Marlon Gastón Ramírez Saverio; Stefani Elizabeth Sánchez Alcívar; Jefferson Saúl Triviño Sánchez

haber administración adicional de 40\% - 100\% de oxígeno en la posición supina. Para los pacientes con una manifestación prolongada de la CPPD se sugiere un tratamiento invasivo.

\section{Farmacoterapia}

Se incluyeron siete ensayos clínicos aleatorios (ECA) con 200 participantes que evaluaron seis fármacos (cafeína, sumatriptán, gabapentina, hidrocortisona, teofilina y hormona adrenocorticotrópica). La cafeína probó ser efectiva para reducir la proporción de participantes con CPPL persistente y que requirieron intervenciones complementarias. La gabapentina, la teofilina y la hidrocortisona también resultaron eficaces para reducir las puntuaciones de intensidad del dolor en comparación con placebo o tratamiento convencional solo, respectivamente. No fue posible un metaanálisis (combinación de los datos) porque todos los ECA incluidos evaluaron fármacos diferentes o resultados diferentes. La falta de información para permitir la evaluación correcta del riesgo de sesgo y los tamaños pequeños de muestra (número de pacientes) de los ECA pueden limitar las conclusiones de esta revisión.

\section{El tratamiento invasivo}

Los pacientes que no responden al tratamiento conservador dentro de las 48 horas se someten a tratamientos invasivos.

\section{Parche de sangre epidural}

El parche Epidural de sangre (EBP) se ha convertido en el “estándar de oro" en el tratamiento de la CPPD. Es un procedimiento relativamente simple; sangre autóloga estéril se inyecta por vía epidural en o cerca del sitio de la punción dural. Los antibióticos profilácticos se 


\section{Tratamiento en pacientes con cefalea postpunción dural}

Vol. 3, núm. 3., (2019)

Gema Nathalye Rodríguez Chica; Marlon Gastón Ramírez Saverio; Stefani Elizabeth Sánchez Alcívar; Jefferson Saúl Triviño Sánchez

usan muy poco. Es ser gravada porque funciona a través de un doble mecanismo: reemplazo de volumen y el sellado de la fuga CSF por la formación de coágulos de sangre local. (Gormley, 1960) fue la primera a la conclusión de que la sangre puede servir como un material de sellado. En su estudio le inyectó 2 - $3 \mathrm{ml}$ de sangre a 7 pacientes (incluyendo a sí mismo) que sufren de CPPD en el sitio de la punción. Su idea más tarde fue apoyada por Di Giovanni y Dunbar. La literatura sugiere volúmenes de inyección de 5 - $30 \mathrm{ml}$ pero la práctica sugiere que el volumen óptimo es aquel en el que se produce presión significativa en la espalda, nalga o extremidades inferiores. Sin embargo, a pesar de que parece un tratamiento altamente eficaz con complicaciones menores, hasta un $30 \%$ de los pacientes experimentan recurrencia de los síntomas y requieren una segunda EBP. Si dos manchas de sangre han sido completó y dolor de cabeza del paciente persiste, a continuación, proyección de imagen es probablemente justificada en la mayoría de las situaciones para confirmar que el diagnóstico correcto se ha hecho, aunque si cada EBP tiene éxito, hay una fuerte posibilidad de que la CPPD es el diagnóstico y la EBP es simplemente fallando a proporcionar un alivio permanente. A pesar de que considera al tratamiento EBP como el estándar de oro es una opción de tratamiento invasivo que no viene sin sus complicaciones; la complicación más común es el dolor de espalda que probablemente es causada debido a la irritación de la raíz nerviosa local y que puede durar hasta 5 días. Otras importantes complicaciones causadas por una inyección accidental de la sangre por vía intratecal, en lugar de por vía epidural, son poco frecuentes e incluyen; infecciones transmitidas por la sangre tales como aracnoiditis o meningitis y varios déficits neurológicos, tales como síndrome de cauda equina o permanente del nervio presa edad. Otras complicaciones reportadas 


\section{Tratamiento en pacientes con cefalea postpunción dural}

Vol. 3, núm. 3., (2019)

Gema Nathalye Rodríguez Chica; Marlon Gastón Ramírez Saverio; Stefani Elizabeth Sánchez Alcívar; Jefferson Saúl Triviño Sánchez

en la literatura incluyen; parálisis del nervio facial, hematoma subdural espinal, mareos, tinnitus, vértigo y ataxia.

\section{Epidural solución salina o coloide}

Es aceptado que un EBP autólogo para el tratamiento de la CPPD puede ser preocupante para algunos pacientes. Por lo tanto, se pensó que una inyección de una solución tal como solución salina sería, en teoría, producir el mismo efecto. Se cree que las soluciones de coloides (dextrano 40\%, hidroxietilalmidón, gelatina fluida modificada) e inyecciones de solución salina en el sitio de la punción lumbar antes de disminuir la pérdida de LCR mediante la restauración de la presión subaracnoidea; Sin embargo, el efecto es transitorio debido a su rápida dispersión. Unos estudios de casos mostraron un alivio del dolor de cabeza en la mayoría de los pacientes, pero con alta tasa (50\%) de recurrencia (Rice \& Dabbs, 1950). Un estudio comparativo de gran tamaño de solución salina con parches de sangre epidurales no ha demostrado la eficacia a largo plazo de la colocación de solución salina epidural. No obstante, parece que las soluciones coloide son favorables en comparación con solución salina epidural; su alto peso molecular y viscosidad producen un efecto más prolongado de la presión epidural y el sello del defecto. No hay suficiente evidencia para apoyar este argumento.

\section{La morfina epidural}

(Eldor, 1990) fueron los primeros en mostrar que la inyección de 3,5 - 4,5 mg de morfina a través de un catéter epidural causado el alivio total del dolor en 6 pacientes. Un estudio publicado posteriormente apoyado esta idea; administración de $3 \mathrm{mg}$ de morfina por vía epidural redujo significativamente CPPD y la necesidad de EBP. 


\section{Tratamiento en pacientes con cefalea postpunción dural}

Vol. 3, núm. 3., (2019)

Gema Nathalye Rodríguez Chica; Marlon Gastón Ramírez Saverio; Stefani Elizabeth Sánchez

Alcívar; Jefferson Saúl Triviño Sánchez

\section{Cola de fibrina}

Otro agente alternativo a la sangre que se ha propuesto para reparar perforación dural es cola de fibrina. La cola de fibrina se compone de dos componentes biológicos, incluyendo solución de trombina y fibrinógeno. El concepto de su interacción se basa en la última etapa de la cascada de coagulación; la trombina convierte el fibrinógeno en monómeros de fibrina que dan lugar a un gel tridimensional en cuestión de segundos.

El pegamento de fibrina se utiliza con mayor frecuencia en neurocirugía para reparar las perforaciones craneales de la duramadre. No hay pruebas suficientes para apoyar el uso de pegamento de fibrina para la CPPD. CPPD con el tratamiento sin incidentes de un parche de sangre epidural. La paciente fue tratada con éxito con la inyección local de la cola de fibrina. Sin embargo, hay un mayor riesgo de infecciones, reacciones inmunes y anafilaxia porque el fibrinógeno es un material biológico a partir de plasma humano combinado.

\section{Cirugía}

Los casos que no responden a la terapia se sugirieron anteriormente se someten a cirugía. Esto es claramente un último recurso de tratamiento.

\section{Nuevo tratamiento sugerido}

Se nos ocurrió un nuevo tratamiento para la CPPD, el tiempo que se debe a una fuga de LCR o debido a la causa menos frecuente: PC. Terapia de oxígeno hiperbárico $(\mathrm{TOH})$ se define por la Sociedad Médica Undersea y hiperbárico (UHMS) como una intervención en la que un endividual respira cerca de $100 \%$ de oxígeno de forma intermitente mientras que dentro de una 


\section{Tratamiento en pacientes con cefalea postpunción dural}

Vol. 3, núm. 3., (2019)

Gema Nathalye Rodríguez Chica; Marlon Gastón Ramírez Saverio; Stefani Elizabeth Sánchez Alcívar; Jefferson Saúl Triviño Sánchez

cámara hiperbárica que se presuriza a presión mayor que el nivel del mar (1 Ambiente Absoluto o ATA). Indicaciones para el tratamiento hiperbárico publicada por la UHMS incluyen: aire o la embolia de gas, el envenenamiento por monóxido de carbono, gangrena gaseosa, isquemia traumática aguda, enfermedad de-compresión, insuficiencias arteriales, anemia grave, absceso intracraneal, infecciones de tejidos blandos-crotizing ne, osteomielitis refractario, retraso en la lesión por radiación, injertos y colgajos com-prometido, lesión ósea térmica aguda y la pérdida de audición neurosensorial súbita idiopática y la presión hidrostática. La elevación de la presión hidrostática comprime los espacios llenos de gas en el cuerpo (la ley de Boyle) y es relevante cuando de gas-balbuceos están presentes en el cuerpo (PC). Por otra parte, la elevación de la O2 presión parcial aumenta la producción de tejidos de especies reactivas de oxígeno (ROS) y especies reactivas de nitrógeno (RNS), por tanto, la promoción de la modulación de cascadas de transducción de intra-celulares, lo que lleva a la síntesis de factores de crecimiento y la promoción de la cicatrización de heridas (Camporesi \& Bosco, 2014). OHB se ha mencionado antes, como una opción terapéutica para PC. (Shih, Tsai, Liao, Wang, \& Hsu, 2015) se presenta un caso de una mujer de 36 años de edad con parada cardiorrespiratoria y coma profundo causado por la epidural PC inducida por la anestesia. Ella fue tratada con éxito con una sesión de TOHB de 120 minutos a 2,8 atm de presión absoluta con 100\% de concentración de oxígenogen. También fue llevado a cabo un pequeño estudio para evaluar los beneficios de la OTHB para el tratamiento de la PC sintomática. Su población de estudio fue en su mayoría pacientes que sufrieron lesiones traumáticas en la cabeza, y se presentan con PC sintomático. A pesar de que su estudio tiene algunas limitaciones, logrado demostrar que OHB en casos selectivos puede conducir a una mejoría clínica y radiológica y puede acortar la estancia hospitalaria. 


\section{Tratamiento en pacientes con cefalea postpunción dural}

Vol. 3, núm. 3., (2019)

Gema Nathalye Rodríguez Chica; Marlon Gastón Ramírez Saverio; Stefani Elizabeth Sánchez Alcívar; Jefferson Saúl Triviño Sánchez

Se cree que la OHB puede ser ofrecida como un mecanismo de doble tratamiento de la CPPD; capear la causa es una fuga del LCR o la presentación menos frecuente de PC. OHB no sólo sirve como un tratamiento exitoso para PC, sino también como un tratamiento adyuvante para la cicatrización de heridas. El fundamento para el tratamiento de heridas crónicas con TOHB se mejora la angiogénesis a través de la proliferación de fibroblastos y síntesis de colágeno. Por otra parte, el TOHB se debe considerar como profilaxis una vez que se sospecha la punción dural, ya que la CPPD es muy probable que desarrollar más adelante.

\section{Conclusiones.}

Dado que la CPPD puede ocurrir después de la punción de la materia de la duramadre, el método más eficaz para disminuir su incidencia es desarrollar técnicas que minimicen la formación del orificio dural durante el bloqueo espinal y que eviten la punción dural inadvertida durante el bloqueo epidural. Con un mejor conocimiento de los factores de riesgo relacionados con la CPPD, es importante que los médicos estén atentos cuando realicen bloqueos espinales o epidurales. En realidad, la punción involuntaria de la duramadre todavía ocurre y la CPPD continúa siendo un problema en estos pacientes. Aunque se han sugerido varias intervenciones profilácticas después de una punción dural inadvertida en función de los factores de riesgo y las inquietudes fisiopatológicas, todavía no hay pruebas suficientes de sus beneficios. Para el tratamiento de la CPPD, un parche de sangre epidural es la modalidad de tratamiento más efectiva, con una alta tasa de éxito. Existen otras varias modalidades de tratamiento para la CPPD, pero aún se necesitan pruebas de alto nivel que respalden su eficacia. En general, se justifica una investigación adicional que incluya grandes ensayos calificados, y se debe prestar atención a estos temas hasta que se disponga de evidencia sustancial. 


\section{Tratamiento en pacientes con cefalea postpunción dural}

Vol. 3, núm. 3., (2019)

Gema Nathalye Rodríguez Chica; Marlon Gastón Ramírez Saverio; Stefani Elizabeth Sánchez Alcívar; Jefferson Saúl Triviño Sánchez

\section{Bibliografía.}

Bradbury, C., Singh, S., Badder, S., Wakely, L., \& Jones, P. (2013). Prevention of Post-Dural Puncture Headache in Parturients: A Systematic Review and Meta-Analysis. Acta Anaesthesiologica Scandinavica, 417-430.

Camporesi, E., \& Bosco, G. (2014). Mechanism of Action of Hyperbaric Oxygen Thera-py. Undersea and Hyperbaric Medicine. 247-252.

Dieterich, M., \& Perkin, G. (1996). Post Lumbar Puncture Headache Syndrome. Neuro-logical Disorders: Course and Treatment. 59-63.

Eldor, J. G. (1990). Epidural Morphine Injections for the Treatment of Post Spinal Headache. Canadian Journal of Anesthesia, 710-711.

Evans, R. (1998). Complications of Lumbar Puncture. . Neurologic Clinics, , 83-105.

Gorelick, P., \& Zych, D. (1987). James Leonard Corning and the Early History of Spinal Puncture. . Neurology, 672-674. .

Gormley, J. (1960). Treatment of Post Spinal Headache. . Anesthesiology, 565-566.

Moayeri, N., H. J., Schaefer, P., \& Zervas, N. (1998). Spinal Dural En-hancement on Magnetic Resonance Imaging Associated with Spontaneous Intracranial Hypotension. Report of Three Cases and Review of the Literature. . Journal of Neurosurgery, 912-918.

Moore, K., Agur, A., \& Dalley, A. (2013). Clinically Oriented Anatomy. . Philadelphia: LWW.

Patin, D., Eckstein, E., Harum, K., \& Pallares, V. (1993). Anatomic and Biomechanical Properties of Human Lumbar Dura Mater. Anesthesia \& Analgesia, 533-540. .

Qunicke, H. (1909). Lumbar Puncture. Diseases of the Nervous System. . New York: Appleton.

Rice, G., \& Dabbs, C. (1950). The Use of Peridural and Subarachnoid Injections of Sa-line Solution in the Treatment of Severe Post Spinal Headache. Anesthesiology, 17-23.

Shih, C.-C., Tsai, S., Liao, W., Wang, J., \& Hsu, C. (2015). Successful Treatment of Epidural Anesthesia-Induced Severe Pneumocephalus by Hyperbaric Oxygen Therapy. American Journal of Emergency Medicine, 1116.

Society., I. H. (07 de 02 de 2001). International Headache Society. Obtenido de http://ihsclassification.org/en/02_klassifikation/03_teil2/07.02.01_nonvascular.html

Turnbull, D., \& Shepherd, D. (2003). Post-Dural Puncture Headache: Pathogenesis, Prevention and Treatment. British Journal of Anesthesia, 718-729. 
Tratamiento en pacientes con cefalea postpunción dural

Vol. 3, núm. 3., (2019)

Gema Nathalye Rodríguez Chica; Marlon Gastón Ramírez Saverio; Stefani Elizabeth Sánchez Alcívar; Jefferson Saúl Triviño Sánchez

Webb, C., Weyker, P., Zhang, L., Stanley, S., Coyle, D., \& Tang, T. (2012). Unintentional dural puncture with a Tuohy needle increases risk of chronic headache. Anesth Analg, 124132.

$$
\text { (9) }(1)(0)
$$

RECONOCIMIENTO-NOCOMERCIAL-COMPARTIRIGUAL

CC BY-NC-SA

ESTA LICENCIA PERMITE A OTROS ENTREMEZCLAR, AJUSTAR Y CONSTRUIR A PARTIR DE SU OBRA CON FINES NO COMERCIALES, SIEMPRE Y CUANDO LE RECONOZCAN LA AUTORÍA Y SUS NUEVAS CREACIONES ESTÉN BAJO UNA LICENCIA CON LOS MISMOS TÉRMINOS. 